\title{
Recurrent metabolic encephalomyopathic crises-rhabdomyolysis-cardiac arrhythmia-intellectual disability syndrome
}

INSERM

\section{Source}

INSERM. (1999). Orphanet: an online rare disease and orphan drug data base. Recurrent metabolic encephalomyopathic crises-rhabdomyolysis-cardiac arrhythmia-intellectual disability syndrome. ORPHA:480864

Recurrent metabolic encephalomyopathic crises-rhabdomyolysis-cardiac arrhythmiaintellectual disability syndrome is a rare, genetic, neurodegenerative disease characterized by episodic metabolic encephalomyopathic crises (of variable frequency and severity which are frequently precipitated by an acute illness) which manifest with profound muscle weakness, ataxia, seizures, cardiac arrhythmias, rhabdomyolysis with myog lobinuria, elevated plasma creatine kinase, hypoglycemia, lactic acidosis, increased acylcarnitines and a disorientated or comatose state. Global developmental delay, intellectual disability and cortical, pyramidal and cerebellar signs develop with subsequent progressive neurodeg eneration causing loss of expressive language and varying degrees of cerebral atrophy. 